\title{
Does negative refraction make a perfect lens?
}

\author{
A. G. Ramm $\dagger$ \\ †Mathematics Department, Kansas State University, \\ Manhattan, KS 66506-2602, USA \\ email: ramm@math.ksu.edu
}

\begin{abstract}
A discussion of a question, studied earlier by V.Veselago in 1967 and by J. Pendry in 2000, is given. The question is: can a slab of the material with negative refraction make a perfect lens? Pendry's conclusion was: yes, it can. Our conclusion is: no, in practice it cannot, because of the fluctuations of the refraction coefficient of the slab. Resolution ability of linear isoplanatic optical instruments is discussed.

key words: Negative refraction, EM waves, wave propagation, resolution ability

MSC: 78A40, 78A45

PACS: 78.20.Ci, 42.30.Wb, 73.20.Mf.
\end{abstract}

\section{Introduction}

Negative refraction has been studied extensively (see [1] and references therein). In [4 the following question is discussed. Assume that a plane electromagnetic (EM) wave $E=e_{2} e^{i\left(k_{1} x+k_{3} z\right)}:=e_{2} e^{i k_{1} x} u_{0}(z), u_{0}(z)=e^{i k_{3} z}$, is incident from the region $z<0$ onto an infinite slab (layer) $0<z<d$, filled with a material with negative refraction (negative index). This means that $\epsilon<0$ and $\mu<0$ in the slab, where $\epsilon$ and $\mu$ are constant dielectric and magnetic parameters. The refractive index

$$
n=(\epsilon \mu)^{1 / 2}=\left(|\epsilon||\mu| e^{2 i \pi}\right)^{1 / 2}=-(|\epsilon||\mu|)^{1 / 2}<0 .
$$

The time dependence is given by the factor $e^{-i \omega t}$ and this factor is omitted throughout. By $\omega>0$ the frequency is denoted. The vectors $\left\{e_{j}\right\}_{j=1}^{3}$ form a Cartesian orthonormal basis of $\mathbb{R}^{3}$. Outside the slab, in the region $z<0$ and $z>d$, we assume that $\epsilon=\epsilon_{0}$ and $\mu=\mu_{0}$, where $\epsilon_{0}=\mu_{0}=1$. It is assumed in [4] that in the slab 
$\epsilon=-1$ and $\mu=-1$, so that $n=-1$ in the region $0 \leq z \leq d$. By $k_{j}$ the components of the wave vector $\vec{k}$ are denoted, $\vec{k}=k_{1} e_{1}+k_{3} e_{3},|\vec{k}|^{2}=\left|k_{1}\right|^{2}+\left|k_{3}\right|^{2}=\omega^{2} \epsilon \mu$. In our case $k_{2}=0$, and $\epsilon \mu>0$. Let us denote the constants $\epsilon$ and $\mu$ in the slab by $\epsilon_{1}$ and $\mu_{1}$.

The governing equations are the Maxwell's equations:

$$
\nabla \times E=i \omega \mu H, \quad \nabla \times H=-i \omega \epsilon E
$$

and the tangential components of the electric and magnetic fields $E_{t}$ and $H_{t}$ are continuous across the interfaces $z=0$ and $z=d$.

Our calculations prove the following conclusion:

If $n=-1$ in the slab, then the image, of a point source, located at the point $(0,0,-f)$, is a point $(0,0,2 d-f)$.

This conclusion agrees with the one obtained earlier in [13] and [4]. In [4] only the propagation of plane waves is discussed. However, the point source is a linear combination of the plane waves (including the evanescent waves), so there is no loss of generality in considering propagation of the plane waves. For example, a well known representation of a point source as a linear combination of the plane waves is the formula:

$$
\frac{e^{i k|x|}}{4 \pi|x|}=\frac{1}{2 \pi i} \iint_{-\infty}^{\infty} \frac{e^{i\left(k_{1} x_{1}+k_{2} x_{2}+x_{3} \sqrt{k^{2}-k_{1}^{2}-k_{2}^{2}}\right)}}{\sqrt{k^{2}-k_{1}^{2}-k_{2}^{2}}} d k_{1} d k_{2}
$$

where $x_{1}=x, x_{2}=y, x_{3}=z$, and $\sqrt{k^{2}-k_{1}^{2}-k_{2}^{2}}>0$ if $k_{1}^{2}+k_{2}^{2}<k^{2}, \sqrt{k^{2}-k_{1}^{2}-k_{2}^{2}}=$ $i \sqrt{\left|k^{2}-k_{1}^{2}-k_{2}^{2}\right|}$ if $k_{1}^{2}+k_{2}^{2}>k^{2}$.

Let us derive the formulas for the electromagnetic field, propagating through the slab, and prove the conclusion, formulated above.

Let us look for a solution to (1) of the form:

$$
E=e_{2} e^{i k_{1} x} u(z), u(z)= \begin{cases}e^{i k_{3} z}+r e^{-i k_{3} z}, & z<0 \\ A e^{i k_{3}^{\prime} z}+B e^{-i k_{3}^{\prime} z}, & 0 \leq z \leq d, \\ t e^{i k_{3} z}, & z>d\end{cases}
$$

where the four coefficients $r, A, B, t$, are to be found from the four boundary conditions:

$$
\begin{gathered}
1+r=A+B, \quad k_{3}(1-r)=\frac{k_{3}^{\prime}}{\mu_{1}}(A-B), \\
A p^{\prime}+\frac{B}{p^{\prime}}=t p, \quad \frac{k_{3}^{\prime}}{\mu_{1}}\left(A p^{\prime}-\frac{B}{p^{\prime}}\right)=t k_{3} p .
\end{gathered}
$$

Here

$$
p^{\prime}:=e^{i k_{3}^{\prime} d}, \quad p:=e^{i k_{3} d}
$$




$$
k_{3}^{\prime}:= \begin{cases}\sqrt{\omega^{2} \epsilon_{1} \mu_{1}-k_{1}^{2}}>0, & k_{1}^{2}<\omega^{2} \epsilon_{1} \mu_{1}, \\ i \sqrt{\left|\omega^{2} \epsilon_{1} \mu_{1}-k_{1}^{2}\right|}, & k_{1}^{2}>\omega^{2} \epsilon_{1} \mu_{1},\end{cases}
$$

and the boundary conditions (3) and (4) come from the continuity of the tangential components of the electric and magnetic fields, that is, $\left[e_{2}, E\right]$ and $\left[e_{3}, H\right]=\frac{\left[e_{3}, \nabla \times E\right]}{i \omega \mu}$, across the planes $z=0$ and $z=d$, respectively. Here $[e, E]$ denotes the vector product of two vectors. The four equations (3), (4) for the four unknowns $r, A, B, t$, can be solved analytically:

$$
\begin{gathered}
r=\gamma \frac{1-p^{\prime 2}}{\gamma^{2} p^{\prime 2}-1}, \quad t=\frac{p^{\prime}}{p} \cdot \frac{\gamma^{2}-1}{\gamma^{2} p^{\prime 2}-1} \\
A=\frac{1-b}{1+b} \cdot \frac{\gamma^{2}-1}{\gamma^{2} p^{\prime 2}-1}, \quad B=\frac{1-b}{2} \cdot \frac{\gamma^{2}-1}{\gamma^{2} p^{\prime 2}-1}
\end{gathered}
$$

where $p^{\prime}$ and $p$ are defined in (5), and

$$
\gamma:=\frac{1-b}{1+b}, \quad b:=\frac{\mu_{1} k_{3}}{\mu_{0} k_{3}^{\prime}} .
$$

If $\epsilon_{1}=\mu_{1}=-1$, then $k_{3}^{\prime}=k_{3}, b=-1, \gamma=\infty$,

$$
r=0, t=e^{-2 i k_{3} d}, A=0, B=1 .
$$

The coefficients $r$ and $t$ are called the reflection and transmission coefficients, respectively. In formulas (12), (21) in [4] by $T$ and $T_{s}$ not the standard transmission coefficients are denoted, but the values $u(z)$ at $z=d$. The values of the field everywhere in the space are given by formulas (2), (5) -(9) .

\section{A discussion of the exact solution}

Let us draw some physical conclusions from the the basic formulas (22), (15)-(9). First, consider the simplest case $\epsilon_{1}=\mu_{1}=-1$. Then formulas (21), (10) hold. These formulas imply that a ray, passing through a point $(0,0,-f), f>0$, with parameters $k_{1}>0, k_{3}>0, k_{2}=0$, will have parameters $\left(k_{1}, 0,-k_{3}\right)$ after passing through the interface $z=0$, and again the parameters $\left(k_{1}, 0, k_{3}\right)$ after passing through the interface $z=d$. In this geometrical argument it is assumed that $d>f$. The ray is the straight line orthogonal to the wave front of the plane wave. The ray issued from the point $(0,0,-f)$ with parameters $\left(k_{1}, 0, k_{3}\right)$, hits the plane $z=0$ at the point $\left(h_{0}, 0,0\right)$, where $h_{0}=f \tan \theta_{0}, \tan \theta_{0}=\frac{k_{1}}{k_{3}}$, and $\theta_{0}$ is the acute angle that the ray makes with $z$-axis. The $z$-axis is perpendicular to the slab. After passing the plane $z=0$, the ray hits the $z$-axis at the point $(0,0, f)$ and then it hits the line $z=d$, 
$y=0$, at the point $\left(-h_{1}, 0,0\right)$, where $h_{1}=(d-f) \tan \theta_{0}$. Finally, after passing the plane $z=d$, the ray hits the $z$-axis at the point $(0,0,2 d-f)$.

The conclusion is :

Regardless of the size of $\theta_{0}$, all the rays, passing through the point $(0,0,-f)$, intersect the $z$-axis at the point $2 d-f, d>f>0$.

This means that the slab with $\epsilon_{1}=\mu_{1}=-1$ acts like a "perfect lens", as is claimed in [13] and [4]. There is no need to discuss separately the case of evanescent waves, i.e., the case when $\omega^{2} \epsilon_{1} \mu_{1}<k_{1}^{2}$. Our derivations are different from those in [4], but the conclusion in the case $\epsilon_{1}=\mu_{1}=-1$ is the same.

However, in [4] he following questions are not discussed:

1) One cannot have $\epsilon=-1$ and $\mu=-1$ exactly due to defects in the material of the slab. Will the above conclusion, (namely, that the image of a bright point $F:=(0,0,-f)$ is a point $(0,0,2 d-f), 0<f<d)$, remain true if $k_{3}^{\prime} \neq k_{3}$ ?

2) Suppose that the slab is not infinite. Will the diffraction from the boundary of the slab invalidate the principal conclusion?

We will not discuss the second question in detail, and restrict ourselves to the following argument. If the size of the slab, which we defined as a maximal radius $R$ of a cylinder, inscribed into the slab, is much larger than the wavelength $\lambda_{0}=\frac{2 \pi}{k_{0}}$, $k_{0}=\omega \sqrt{\epsilon_{0} \mu_{0}}, R \gg \lambda_{0}$ and $R \gg l$, where $l$ is the size of the object, then one may neglect the diffraction of light at the boundary of the slab, provided that the object is located near z-axis, far away from the boundary of the slab.

The first question we discuss in more detail. The aim of our discussion is to conclude that if $\epsilon_{1}$ or $\mu_{1}$ differ slightly from -1 , so that $n$ is not equal to -1 exactly, then the image of a point $(0,0,-f)$ is no longer a point. More precisely, not all the rays, passing throught the point $F:=(0,0,-f)$, pass through the common point of the $z$-axis after the plane $z=d$. It turns out that the point of itersection of the ray, passing through $F$ at the angle $\theta_{0}$ with the $z$-axis in the region $z>d$, will depend on $\theta_{0}$ if $\left|k_{3}^{\prime}\right| \neq\left|k_{3}\right|$. Assume, for example, that $\mu_{1}=-1+\delta_{\mu}, \epsilon_{1}=-1+\delta_{\epsilon}, \delta_{\mu}=\delta_{\epsilon}=\delta$, where $\delta>0$ is a small number. Then

$$
\begin{aligned}
k_{3}^{\prime} & =\sqrt{\omega^{2}\left(-1+\delta_{\mu}\right)\left(-1+\delta_{\epsilon}\right)-k_{1}^{2}} \approx \sqrt{\omega^{2}-k_{1}^{2}-\omega^{2}\left(\delta_{\mu}+\delta_{\epsilon}\right)} \\
& =k_{3} \sqrt{1-\frac{2 \omega^{2} \delta}{k_{3}^{2}}} \approx k_{3}-\frac{\omega^{2} \delta}{k_{3}}, \quad k_{3}=\sqrt{\omega^{2}-k_{1}^{2}} .
\end{aligned}
$$

Here we have neglected the term of order $O\left(\delta^{2}\right)$, and assumed that $\left|k_{3}\right| \gg \delta>0$. Thus $\frac{k_{3}^{\prime}}{k_{3}}=1-\delta_{1}$, where $\delta_{1}:=\frac{\omega^{2} \delta}{k_{3}}$.

If $k_{3}=O(\delta)$ then $\left|k_{3}^{\prime}\right|=O\left(\delta^{1 / 2}\right)$. In this case $\frac{k_{3}^{\prime}}{k_{3}}$ can take rather arbitrary values depending on the ratio $\frac{\delta}{\left|\omega^{2}-k_{1}^{2}\right|}$, and therefore the deviation of the corresponding rays from the point $(0,0,2 d-f)$ may be much greater than $O(\delta)$. 
If $\left|k_{3}\right| \gg \delta$, then we repeat the geometrical arguments given for the case $k_{3}^{\prime}=k_{3}$, and get the following conclusions: the ray, passing through the point $F:=(0,0,-f)$ and having angle $\theta_{0}$ with the $z$-axis, hits the plane $z=0$ at the point $\left(h_{0}, 0,0\right)$, $h_{0}=f \tan \theta_{0}$, then it hits the $z$-axis at the point $\left(0,0, f^{\prime}\right)$, where $\tan \theta^{\prime}=\frac{h_{0}}{f^{\prime}}=\frac{k_{1}}{k_{3}}$, so $f^{\prime}=h_{0} \cot \theta^{\prime}$, and then it hits the plane $z=d$ at the point $\left(-h_{1}^{\prime}, 0,0\right)$, where

$\frac{h_{1}^{\prime}}{d-f^{\prime}}=\tan \theta^{\prime}=\frac{k_{1}}{k_{3}^{\prime}}$. Finally, after passing the plane $z=d$, this ray hits the $z$-axis at the point $\left(0,0, d+z^{\prime}\right)$, where $z^{\prime}=h_{1}^{\prime} \cot \theta_{0}$. Therefore,

$$
d+z^{\prime}=d+\left(d-f \tan \theta_{0} \cot \theta^{\prime}\right) \tan \theta^{\prime} \cot \theta_{0}=d\left(1+\tan \theta^{\prime} \cot \theta_{0}\right)-f .
$$

One has $\tan \theta_{0}=\frac{k_{1}}{k_{3}}, \tan \theta^{\prime}=\frac{k_{1}}{k_{3}^{\prime}}$, so $\tan \theta^{\prime} \cot \theta_{0}=\frac{k_{3}}{k_{3}^{\prime}}=\frac{1}{1-\delta_{1}}$.

Conclusion: The ray, passing through the point $F=(0,0,-f)$ under the angle $\theta_{0}$ with $z$-axis, hits the $z$-axis, after passing the slab, at the point whose coordinate $\left(0,0, d-f+d \tan \theta^{\prime} \cot \theta_{0}\right)$ depends on the angle $\theta_{0}$. Therefore, the image of a point source, located at the point $F$, will not be a point.

At a fixed $\omega>0$ the deviation of the image of a bright point depends on the deviation of $\frac{k_{3}}{k_{3}^{\prime}}$ from 1 . This implies a possibility to make changes in $\epsilon_{1}$, if it is practically easier than to make changes in $\mu_{1}$, in order to compensate for the deviations of $\mu_{1}$ from -1 .

\section{On the resolution ability of linear isoplanatic optical instruments}

Following L. Mandel'shtam [2], assume that a linear isoplanatic optical instrument $\mathcal{S}$ creates an image of an object as follows. A bright point (a point source), is located on a plane $P_{1}$ at a point $y \in \mathbb{R}^{3}$, and the focal plane is located to the left of $\mathcal{S}$. The bright point produces an image $h(x, y)=h(x-y)$ on the image plane $P_{2}, x \in P_{2}$, and $P_{2}$ is the plane to the right of $\mathcal{S}$. The function $h=h(x)$ is called the scattering function of the instrument $\mathcal{S}$. The instrument is called isoplanatic if $h(x, y)=h(x-y)$. The image of an object $f(x)$, located in the plane $P_{1}$ is

$$
u(x)=\int_{P_{1}} h(x-y) f(y) d y, \quad x \in P_{2} .
$$

The problem of the resolution ability of $\mathcal{S}$ in Rayleigh's formulation is stated as follows:

Given two signals, $f_{1}=\delta(x-a), f_{2}=\delta(x+a)$, where $\delta(x)$ is the delta-function and $a$ is a vector, $|a|$ is small, will the function $\frac{h(x+a)+h(x-a)}{2}$ have one or two maxima near $x=0$ ? If two maxima can be detected, then, according to Rayleigh, the instrument $\mathcal{S}$ can discriminate between the two point sources the distance between 
which is $2 a$.

This criterion of the resolution ability is very limited: the class of objects to be discriminated consists of two point sources. Rayleigh's conclusion is : one cannot discriminate between two point sources if $a \sim \frac{\lambda}{2}$, because the width of the function $h(x)$ is of the order of $\lambda$, where $\lambda$ is the wavelength.

In [5]-[12] the resolution ability problem was examined in detail. In [8] a new definition of resolution ability of a linear instrument is given. This definition allows for a very wide class of the input signals, not only for two point sources (bright points), as in Rayleigh's definition. It takes into account the noise on the image plane, and it takes into account the properties of the procedure that reconstructs the input signal from its noisy image. The principal conclusion in [8] is :

The resolution ability in Rayleigh's sense can be increased without a limit by apodization.

In other words, the scattering function of a linear isoplanatic optical instrument can be made by apodization as close as one wishes to a delta-function on any fixed finite subdomain of the image plane. This conclusion is justified as follows. The scattering function is $h(x)=\int_{D} j(y) e^{-i x \cdot y} d y$, where $x \cdot y$ is the dot product of vectors, and $D$ is a finite region, it is the output pupil of the optical instrument $\mathcal{S}$, (see [3], Sec. 5.3), and $j(y)$ is the field distribution on the output pupil $D$. If one puts a mask on the output pupil $D$, then the field $j(y)$ is transformed into $j(y) n(y)$, where $n(y)$ is the refraction coefficient of the mask. Putting the mask on $D$ results in the change of $h$ : the new $h=h_{n}:=\int_{D} j(y) n(y) e^{-i x \cdot y} d y$. It is proved in [5], 6], 7], that, given a bounded domain $\Delta$ on the image plane, one can find a sequence of functions $f_{m}(y)$ such that the sequence $\delta_{m}(x):=\int_{D} f_{m}(y) e^{-i x \cdot y} d y$ is a delta-sequence on $\Delta$, that is $\lim _{m \rightarrow \infty} \int_{\Delta} \delta_{m}(x-s) \phi(s) d s=\phi(x)$ for any smooth function $\phi$ vanishing outside $\Delta$. Moreover, the sequence $f_{m}(y)$ is given explicitly, analytically, in [6] and in [11], see also [12, p.211]. Therefore one can choose $n_{m}(y)$ so that $j(y) n_{m}(y)=f_{m}(y)$, and then the correspionding scattering functions $h_{m}(x)$ form a delta-sequence on the finite domain $\Delta$, so that the width of $h_{m}(x)$ can be made as small as one wishes.

By the Rayleigh criterion, this means that, for sufficiently large $m$ the resolution ability (in Rayleigh's sense) can be increased without a limit. In this argument we did not take into account the noise in the image. It is proved in [8] that it is possible to increase the resolution ability (in the Rayleigh's sense) of a linear isoplanatic optical system without a limit by apodization even in the presence of noisy background in the image plane. The noise is assumed to be independent identically distributed with zero mean value and an arbitrary large but fixed finite variance. Our arguments are valid under the assumption that the classical Maxwell's equations are valid. We do not discuss quantum effects in this paper.

The author thanks Prof. O.L. Weaver for discussions. 


\section{References}

[1] V. Agranovich, Yu. Gartstein, Spatial dispersion and negative refraction of light, Physics-Uspekhi, 176,N10,(2006),1051-1068.

[2] L. Mandel'shtam, lectures on optics, relativity and quantum mechanics, Nauka, Moscow, 1972.

[3] E. O’Neill, Introduction to statistical optics, Addison-Wesley, London, 1963.

[4] J. Pendry, Negative refraction makes a perfect lens, Phys. Rev. Lett., 85,N18,(2000), 3966-3969.

[5] A.G. Ramm, Apodization theory, Optics and Spectroscopy, 27, (1969), 508-514.

[6] A.G. Ramm, Apodization theory II, Optics and Spectroscopy, 29, (1970), 390-394.

[7] A.G. Ramm, Increasing of the resolution ability of the optical instruments by means of apodization, Optics and Spectroscopy, 29, (1970), 594-599.

[8] A.G. Ramm, On resolution ability of optical systems, Optics and Spectroscopy, 29, (1970), 794-798.

[9] A.G. Ramm, Discrimination of the random fields in noises, Probl. peredaci informacii (Problems of information transmission), 9, (1973), 22-35.

[10] A.G. Ramm, S. Rodionov, Optimization of the resolution ability, Optics and Spectroscopy, 42, (1977), 540-545.

[11] A.G. Ramm, Approximation by entire functions, Mathematics, Izv. vusov, 10, (1978), 72-76.

[12] A.G. Ramm, Theory and applications of some new classes of integral equations, Springer-Verlag, New York, 1980.

[13] V.Veselago,The electrodynamics of media with negative $\epsilon$ and $\mu$, Sov. Phys. Uspekhi, 92, (1967), 517-526. 